九州大学学術情報リポジトリ

Kyushu University Institutional Repository

\title{
Non-linear algebraic differential equations satisfied by certain family of elliptic functions
}

Wakayama, Masato

Faculty of Mathematics, Kyushu University

Yamamoto, Keitaro

Faculty of Mathematics, Kyushu University

http://hdl. hand le. net/2324/14239

出版情報：The Ramanujan journal. 30 (2)，pp.173-186，2013-02. Springer US バージョン:

権利関係: (C) Springer Science+Business Media, LLC 2012 


\section{Preprint Series}

Kyushu University

The Grobal COE Program

Math-for-Industry Education \& Research Hub

Non-linear algebraic differential equations satisfied by certain family of elliptic functions

\section{Wakayama \& K. Yamamoto \\ MI 2009-17}

( Received April 30, 2009 )

Faculty of Mathematics

Kyushu University

Fukuoka, JAPAN 


\title{
Non-linear algebraic differential equations satisfied by certain family of elliptic functions
}

\author{
Masato Wakayama * and Keitaro Yamamoto
}

April 27, 2009

\begin{abstract}
The paper $[\mathrm{KW}]$ studied a family of elliptic functions defined by certain $q$-series. This family, in particular, contains the Weierstrass $\wp$-function. In this paper, we prove that elliptic functions in this family satisfy certain non-linear algebraic differential equations whose coefficients are essentially given by rational functions of the first few Eisenstein series of the modular group.
\end{abstract}

Keywords: elliptic functions, $q$-series, Weierstrass $\wp$-function, Eisenstein series, Lambert series.

2000 Mathematical Subject Classification: 11M36, 33E05

\section{Introduction}

In the paper $[\mathrm{KW}]$, Kurokawa and the first author studied the q-series $L_{\ell}(x)(x \in \mathbb{C})$ defined by

$$
L_{\ell}(x):=\sum_{n \in \mathbb{Z}} \frac{q^{\frac{1}{2}(n+x) \ell}}{[n+x]_{q}^{\ell}} \quad(\ell=1,2,3, \ldots) .
$$

Here we put $[\beta]_{q}:=\frac{q^{\beta}-1}{q-1}$ for $\beta \in \mathbb{C}$. Throughout the present paper, we assume that $q>1$. The series $L_{\ell}(x)$ converges absolutely and uniformly in $x$, whence defines an elliptic function for the period lattice $\mathbb{Z} \cdot 1+\mathbb{Z} \cdot \frac{2 \pi i}{\log q}$ when $\ell$ is even, while $\mathbb{Z} \cdot 1+\mathbb{Z} \cdot \frac{4 \pi i}{\log q}$ when $\ell$ is odd. Precisely, $L_{\ell}$ has the following properties.

$$
\left\{\begin{array}{l}
L_{\ell}(x+1)=L_{\ell}(x), \\
L_{\ell}\left(x+\frac{2 \pi i}{\log q}\right)=(-1)^{\ell} L_{\ell}(x), \\
L_{\ell}(-x)=(-1)^{-\ell} L_{\ell}(x) .
\end{array}\right.
$$

It is shown in $[\mathrm{KW}]$ that the series $L_{2}(x)$ gives essentially the Weierstrass $\wp$-function and hence, in particular, satisfies the non-linear algebraic differential equation. An algebraic differential equation for $L_{1}$ is also derived. Beside this fact, it was proved that $L_{2 \ell-1} \in \mathbb{C}\left[L_{1}\right]$ and $L_{2 \ell} \in \mathbb{C}\left[L_{2}\right]$. In fact, $L_{2 \ell-1}$ (resp. $L_{2 \ell}$ ) is expressed as a polynomial in $L_{1}$ (resp. $L_{2}$ ) of degree $2 \ell-1$ (resp. $\ell$ ). Moreover, one may replace the fields of the coefficients $\mathbb{C}$ by the ring $\mathbb{Q}(q)$ of Laurent polynomials over $\mathbb{Q}$. From these facts, it was conjectured in $[\mathrm{KW}]$ that the elliptic function $L_{\ell}(x)$ satisfies a non-linear algebraic differential equation for each positive integer $\ell$ with coefficients in $\mathbb{Q}(q)$.

The purpose of this paper is to prove this conjecture affirmatively and give some precise information about the coefficients of the differential equation. Actually, for instance, we will show that the coefficients in the even $\ell$ are given by rational functions of $(q-1)^{2},(\log q)^{2}$ and the Lambert series $A_{\ell}(q)(\ell=1,3,5)$ over $\mathbb{Q}$. We note that $A_{2 \ell+1}(q)$ gives essentially the Fourier series expansion of the Eisenstein series $E_{2 \ell}(z)$ for the modular group $S L_{2}(\mathbb{Z})$ if we put $q=e^{-2 \pi i z}(\operatorname{Im}(\mathrm{z})>0)[\mathrm{L}]$.

\footnotetext{
*Work in part supported by Grant-in-Aid for Scientific Research (B) No. 21340011.
} 


\section{Basic facts on $L_{\ell}$}

Let us recall first the basic facts in $[\mathrm{KW}]$, the algebraic differential equations and the Laurent expansion of the functions $L_{1}(x)$ and $L_{2}(x)$.

The differential equations for $L_{1}$ and $L_{2}$ are described as follows.

Lemma 2.1. Define the Lambert series $B_{\ell}(q)$ by

$$
B_{\ell}(q):=\sum_{m=0}^{\infty} \frac{\left(m+\frac{1}{2}\right)^{\ell}}{q^{m+\frac{1}{2}}-1} .
$$

Then we have

$$
\left(L_{1}^{\prime}\right)^{2}=\frac{e_{2}}{e_{1}} L_{1}^{4}+e_{2}\left(e_{3}+\frac{1}{4}\right) L_{1}^{2}+e_{1} e_{2} e_{4},
$$

where

$$
\left\{\begin{array}{l}
e_{1}=(q-1)^{2} \\
e_{2}=(\log q)^{2} \\
e_{3}=12 B_{1}(q) \\
e_{4}=\frac{9}{4}\left(\frac{1}{12}+4 B_{1}(q)\right)^{2}-\frac{1}{2}\left(\frac{1}{12}+4 B_{1}(q)\right)-\frac{1}{4}\left(\frac{7}{240}-16 B_{3}(q)\right) .
\end{array}\right.
$$

Lemma 2.2. Define the Lambert series $A_{\ell}(q)$ by

$$
A_{\ell}(q):=\sum_{m=1}^{\infty} \frac{m^{\ell}}{q^{m}-1}=\sum_{j=1}^{\infty} \sigma_{\ell}(j) q^{-j} \quad\left(\sigma_{\ell}(j)=\sum_{m \mid \ell} m^{j}\right) .
$$

Then we have

$$
\left(L_{2}^{\prime}\right)^{2}=\frac{4 d_{2}}{d_{1}} L_{2}^{3}+d_{2}\left(6 d_{3}+1\right) L_{2}^{2}+4 d_{1} d_{2} d_{4} L_{2}+d_{1}^{2} d_{2} d_{5},
$$

where

$$
\left\{\begin{array}{l}
d_{1}=(q-1)^{2}, \\
d_{2}=(\log q)^{2} \\
d_{3}=-4 A_{1}(q) \\
d_{4}=-A_{1}(q)+12 A_{1}^{2}(q)-5 A_{3}(q), \\
d_{5}=\frac{1}{432}\left(1-24 A_{1}(q)\right)^{3}-\frac{1}{144}\left(1-24 A_{1}(q)\right)\left(1+240 A_{3}(q)\right)-\frac{1}{216}\left(1-504 A_{5}(q)\right) .
\end{array}\right.
$$

Remark 2.3. The series $L_{2}$ is essentially the Wierstrass $\wp$-function. Actually, if we define

$$
\Omega_{2}(x):=(q-1)^{-2} L_{2}(x)+\frac{1}{12}\left(1-24 A_{1}(q)\right),
$$

we see that

$$
\left(\Omega_{2}^{\prime}\right)^{2}=\alpha_{0} \Omega_{2}^{3}-\alpha_{1} \Omega_{2}-\alpha_{2}
$$

where

$$
\left\{\begin{array}{l}
\alpha_{0}=4(\log q)^{2} \\
\alpha_{1}=(\log q)^{2} \frac{1}{12}\left(1+240 A_{3}(q)\right) \\
\alpha_{2}=(\log q)^{2} \frac{1}{216}\left(1-504 A_{5}(q)\right)
\end{array}\right.
$$


Remark 2.4. Note that the Eisenstein series $E_{2 \ell}(z)$ (see $\left.[\mathrm{L}]\right)$ is given by

$$
E_{2 \ell}(z):=\frac{1}{2} \sum_{(c, d)=1}(c z+d)^{-2 \ell}=1+\frac{2}{\zeta(1-2 \ell)} A_{2 \ell-1}(q) \quad\left(q=e^{-2 \pi i z}, \operatorname{Im}(\mathrm{z})>0\right) .
$$

Also, the series $B_{\ell}(q)$ is expressed as

$$
B_{\ell}(q)=2^{-\ell}\left[A_{\ell}\left(q^{\frac{1}{2}}\right)-2^{\ell+1} A_{\ell}(q)\right] .
$$

In the proof of the existence of the differential equations satisfied by $L_{1}(x)$ and $L_{2}(x)$ in [KW], we have seen the following Laurent expansion of these functions.

\section{Lemma 2.5.}

$$
\begin{gathered}
(q-1)^{-1} L_{1}(x)=-2 \sum_{l=0}^{\infty} \frac{B_{2 l+1}(q)}{(2 l+1) !}(x \log q)^{2 l+1}+\frac{1}{x \log q}-\frac{x \log q}{24}+O\left(x^{3}\right) \\
(q-1)^{-2} L_{2}(x)=2 \sum_{l=0}^{\infty} \frac{A_{2 l+1}(q)}{(2 l) !}(x \log q)^{2 l}+\frac{1}{(x \log q)^{2}}-\frac{1}{12}+O\left(x^{2}\right) .
\end{gathered}
$$

Using these Laurent expansions, we have the following relation between $L_{1}(x)$ and $L_{2}(x)$ which was suggested by Robin Chapman [C].

\section{Proposition 2.6.}

$$
L_{2}(x)=L_{1}(x)^{2}+(q-1)^{2}\left(2 A_{1}(q)+4 B_{1}(q)\right) .
$$

Proof. Put $F(x):=L_{2}(x)-L_{1}(x)^{2}$. Then, from the lemma above one obtains

It follows that

$$
\begin{aligned}
(q-1)^{-1} L_{1}(x) & =\frac{1}{\log q} \frac{1}{x}-(\log q)\left(2 B_{1}(q)+\frac{1}{24}\right) x+O\left(x^{3}\right), \\
(q-1)^{-2} L_{2}(x) & =\frac{1}{(\log q)^{2}} \frac{1}{x^{2}}+\left(2 A_{1}(q)-\frac{1}{12}\right)+O\left(x^{2}\right) .
\end{aligned}
$$

$$
F(x)=(q-1)^{2}\left(2 A_{1}(q)+4 B_{1}(q)\right)+O\left(x^{2}\right)
$$

Also, since

$$
L_{\ell}(x+1)=L_{\ell}(x) \quad \text { and } \quad L_{\ell}\left(x+\frac{2 \pi i}{\log q}\right)=(-1)^{\ell} L_{\ell}(x),
$$

we immediately find that $F(x+1)=F(x)$ and $F\left(x+\frac{2 \pi i}{\log q}\right)=F(x)$. This implies that $F(x)$ is an (continuous) elliptic function with period $\mathbb{Z} \cdot 1+\mathbb{Z} \cdot \frac{2 \pi i}{\log q}$. It follows hence from the Liouville theorem that $F(x)$ is equal to a constant $F(0)=(q-1)^{2}\left(2 A_{1}(q)+4 B_{1}(q)\right)$. This proves the proposition.

Corollary 2.7. We have $L_{\ell} \in \mathbb{C}\left[L_{1}\right]$. If we write $L_{\ell}=p_{\ell}\left(L_{1}\right)$, $p_{\ell}$ is a monic polynomial of degree $\ell$ and satisfies $p_{\ell}(-x)=(-1)^{\ell} p_{\ell}(x)$.

Proof. Since $L_{1} \in \mathbb{C}\left[L_{1}\right]$ (of degree $2 \ell-1$ ) and $L_{2} \in \mathbb{C}\left[L_{2}\right]$ (of degree $\ell$ ), it follows from Proposition 2.6 that $f_{\ell}$ is of degree $\ell$. Furthermore, one has

$$
\begin{aligned}
p_{\ell}\left(-L_{1}(x)\right) & =p_{\ell}\left(L_{1}(-x)\right) \\
& =L_{\ell}(-x)=(-1)^{\ell} L_{\ell}(x) \\
& =(-1)^{\ell} p_{\ell}\left(L_{1}(x)\right)
\end{aligned}
$$

whence the desired relation follows immediately. The property that $p_{\ell}$ is monic follows from the discussion developed in the proof of Proposition 3.2 and Proposiotn 3.4 in the subsequent section. 


\section{Algebraic Differential equations}

\subsection{Differential equations for $L_{2 \ell}$}

We prove the derivatives $L_{2 \ell}^{(2 k)}$ and $\left(L_{2 \ell}^{(2 k+1)}\right)^{2}(k=0,1,2, \ldots)$ of $L_{2 \ell}$ can be expressed as polynomilas in $L_{2}$. To show this, we need the following difference-differential equation satisfied by $L_{\ell}$ 's $[\mathrm{KW}]$.

\section{Lemma 3.1.}

$$
L_{\ell}^{\prime \prime}=\frac{(\log q)^{2}}{4} \cdot \ell^{2} L_{\ell}+\frac{(\log q)^{2}}{(q-1)^{2}} \cdot \ell(\ell+1) L_{\ell+2} .
$$

We have then the following.

Proposition 3.2. There exist constants

$$
a_{i}(k), b_{i}(k) \in \mathbb{Q}\left(d_{1}, d_{2}, d_{3}, d_{4}, d_{5}\right) \quad\left(a_{\ell+k}(k) \neq 0, b_{2 \ell+2 k+1}(k) \neq 0\right)
$$

such that the functions $L_{2 \ell}^{(2 k)}$ and $\left(L_{2 \ell}^{(2 k+1)}\right)^{2}$ are expressed by the polynomials of $L_{2}$ as

$$
\begin{aligned}
L_{2 \ell}^{(2 k)} & =\sum_{i=0}^{\ell+k} a_{i}(k) L_{2}^{i}, \quad\left(a_{i}(0)=1\right), \\
\left(L_{2 \ell}^{(2 k+1)}\right)^{2} & =\sum_{i=0}^{2 \ell+2 k+1} b_{i}(k) L_{2}^{i} .
\end{aligned}
$$

Proof. By Lemma 3.1 and (2.1) we easily obtain

$$
L_{\ell}^{\prime \prime}=\frac{d_{2}}{4} \cdot \ell^{2} L_{\ell}+\frac{d_{2}}{d_{1}} \cdot \ell(\ell+1) L_{\ell+2}
$$

and

$$
\left(L_{2}^{\prime}\right)^{2}=\frac{4 d_{2}}{d_{1}} L_{2}^{3}+d_{2}\left(6 d_{3}+1\right) L_{2}^{2}+4 d_{1} d_{2} d_{4} L_{2}+d_{1}^{2} d_{2} d_{5}
$$

Differentiating (3.2) we have

$$
L_{2}^{\prime \prime}=\frac{6 d_{2}}{d_{1}} L_{2}^{2}+d_{2}\left(6 d_{3}+1\right) L_{2}+2 d_{1} d_{2} d_{4}
$$

We first prove the former assertion for $k=0$ by induction on $\ell$. Let us assume that the assertion is true for $2 \ell$ :

$$
L_{2 \ell}=\sum_{i=0}^{\ell} a_{i} L_{2}^{i}, \quad\left(a_{i} \in \mathbb{Q}\left(d_{1}, \cdots, d_{5}\right), a_{\ell}=1\right) .
$$

Then, since $L_{2 \ell}^{\prime}=\left\{\sum_{i=1}^{\ell} i a_{i} L_{2}^{i-1}\right\} L_{2}^{\prime}$, one gets

$$
L_{2 \ell}^{\prime \prime}=\left\{\sum_{i=2}^{\ell} i(i-1) a_{i} L_{2}^{i-2}\right\}\left(L_{2}^{\prime}\right)^{2}+\left\{\sum_{i=1}^{\ell} i a_{i} L_{2}^{i-1}\right\} L_{2}^{\prime \prime} .
$$

It follows that $L_{2 \ell}^{\prime \prime}$ can be written by a polynomila of $L_{2}$ as

$$
L_{2 \ell}^{\prime \prime}=\sum_{i=0}^{\ell+1} b_{i} L_{2}^{i}, \quad\left(b_{i} \in \mathbb{Q}\left(d_{1}, \cdots, d_{5}\right)\right.
$$


with

$$
b_{\ell+1}=\ell(\ell-1) a_{\ell} \frac{4 d_{2}}{d_{1}}+\ell a_{\ell} \frac{6 d_{2}}{d_{1}}=2 \ell(2 \ell+1) \frac{d_{2}}{d_{1}} \neq 0 .
$$

Now replacing $\ell$ by $2 \ell$ in (3.1) we see that

$$
L_{2 \ell}^{\prime \prime}=d_{2} \ell^{2} L_{2 \ell}+\frac{d_{2}}{d_{1}} 2 \ell(2 \ell+1) L_{2 \ell+2} .
$$

Hence we see that

$$
L_{2 \ell+2}=\sum_{i=0}^{\ell+1} c_{i} L_{2}^{i}, \quad\left(c_{i} \in \mathbb{Q}\left(d_{1}, \cdots, d_{5}\right)\right)
$$

with

$$
c_{\ell+1}=\frac{b_{\ell+1}}{\frac{d_{2}}{d_{1}} 2 \ell(2 \ell+1)}\left(=a_{\ell}\right)=1 .
$$

This proves the assertion for $k=0$.

We next prove the assertion for general $k$ by induction. Suppose that $L_{2 \ell}^{(2 k)}$ can be written as

$$
L_{2 \ell}^{(2 k)}=\sum_{i=0}^{\ell+k} a_{i} L_{2}^{i}, \quad\left(a_{i} \in \mathbb{Q}\left(d_{1}, \cdots, d_{5}\right), a_{\ell+k} \neq 0\right) .
$$

Then, taking derivatives twice we have

$$
L_{2 \ell}^{(2 k+2)}=\left\{\sum_{i=2}^{\ell+k} i(i-1) a_{i} L_{2}^{i-2}\right\}\left(L_{2}^{\prime}\right)^{2}+\left\{\sum_{i=1}^{\ell+k} i a_{i} L_{2}^{i-1}\right\} L_{2}^{\prime \prime} .
$$

From this it follows that

$$
L_{2 \ell}^{(2 k+2)}=\sum_{i=0}^{\ell+k+1} b_{i} L_{2}^{i}, \quad\left(b_{i} \in \mathbb{Q}\left(d_{1}, \cdots, d_{5}\right)\right)
$$

with

$$
b_{\ell+k+1}=2(\ell+k)(2 \ell+2 k+1) a_{\ell+k} \frac{d_{2}}{d_{1}} \neq 0 .
$$

This proves the first equation of the lemma for $L_{2 \ell}^{(2 k)}$. Furthermore, differentiating this equation and taking square we observe

$$
\left(L_{2 \ell}^{(2 k+1)}\right)^{2}=\left(\sum_{i=1}^{\ell+k} i a_{i} L_{2}^{i-1}\right)^{2}\left(L_{2}^{\prime}\right)^{2} .
$$

From this expression, we see that $\left(L_{2 \ell}^{(2 k+1)}\right)^{2}$ is expressed by a polynomial in $L_{2}$ as

$$
\left(L_{2 \ell}^{(2 k+1)}\right)^{2}=\sum_{i=0}^{2 \ell+2 k+1} b_{i} L_{2}^{i}, \quad\left(b_{i} \in \mathbb{Q}\left(d_{1}, \cdots, d_{5}\right)\right)
$$

with

$$
b_{2 \ell+2 k+1}=(\ell+k)^{2} a_{\ell+k}^{2} \frac{4 d_{2}}{d_{1}} \neq 0 .
$$

This completes the proof of the proposition. 
We now get a non-linear algebraic differential equation for $L_{2 \ell}$.

Theorem 3.3. The function $L_{2 \ell}(\ell \geq 1)$ satisfies the following differential equation:

$$
\sum_{i=0}^{\ell} \alpha_{i} L_{2 \ell}^{(2 i)} L_{2 \ell}^{(4 \ell-2 i)}+\sum_{i=0}^{3 \ell-1} \beta_{i} L_{2 \ell}^{(2 i)}+\gamma=0 \quad\left(\alpha_{i}, \beta_{i}, \gamma \in \mathbb{Q}\left(d_{1}, \cdots, d_{5}\right)\right) .
$$

Proof. By Proposition 3.2 one finds that the degree of the polynomial $L_{2 \ell}^{(2 i)} L_{2 \ell}^{(4 \ell-2 i)}$ in $L_{2}$ is calculated as $(\ell+i)+(\ell+2 \ell-i)=4 \ell$ for all $0 \leq i \leq \ell$. In particular, since the degree of the polynomials $L_{2 \ell}^{\prime \prime} L_{2 \ell}^{(4 \ell-2)}$, $L_{2 \ell} L_{2 \ell}^{(4 \ell)}, L_{2 \ell}^{(6 \ell-2)}, \cdots, L_{2 \ell}$ are $4 \ell, 4 \ell, 4 \ell-1, \cdots, \ell$, respectively, if one eliminates the leading coefficients successively one obtains

$$
f_{1}:=L_{2 \ell}^{\prime \prime} L_{2 \ell}^{(4 \ell-2)}+a_{1,1} L_{2 \ell} L_{2 \ell}^{(4 \ell)}+a_{1,2} L_{2 \ell}^{(6 \ell-2)}+\cdots+a_{1,3 \ell+1} L_{2 \ell}=\sum_{i=0}^{\ell-1} b_{1, i} L_{2}^{i}
$$

for some coefficients $a_{1, j}, b_{1, i} \in \mathbb{Q}\left(d_{1}, \cdots, d_{5}\right)$. Similarly, for $k=2, \ldots, \ell$, there exist $a_{i, j}, b_{k, l} \in \mathbb{Q}\left(d_{1}, \cdots, d_{5}\right)$ one has

$$
f_{k}:=L_{2 \ell}^{(2 k)} L_{2 \ell}^{(4 \ell-2 k)}+a_{k, 1} L_{2 \ell} L_{2 \ell}^{(4 \ell)}+a_{i, 2} L_{2 \ell}^{(6 \ell-2)}+\cdots+a_{k, 3 \ell+1} L_{2 \ell}=\sum_{i=0}^{\ell-1} b_{k, i} L_{2}^{i} .
$$

Write these relations as

$$
\left(\begin{array}{c}
f_{1} \\
f_{2} \\
\vdots \\
f_{\ell}
\end{array}\right)=\left(\begin{array}{cccc}
b_{1,0} & b_{1,1} & \cdots & b_{1, \ell-1} \\
b_{2,0} & \ddots & & b_{2, \ell-1} \\
\vdots & & \ddots & \vdots \\
b_{\ell, 0} & \cdots & \cdots & b_{\ell, \ell-1}
\end{array}\right)\left(\begin{array}{c}
1 \\
L_{2} \\
\vdots \\
L_{2}^{\ell-1}
\end{array}\right)
$$

Hence, there exists an $\ell \times \ell$ matrix $A$ with coefficients in $\mathbb{Q}\left(d_{1}, \cdots, d_{5}\right)$ such that

$$
A\left(\begin{array}{c}
f_{1} \\
f_{2} \\
\vdots \\
f_{\ell}
\end{array}\right)=\left(\begin{array}{cccc}
c_{1,0} & 0 & \cdots & 0 \\
c_{2,0} & \ddots & \ddots & \vdots \\
\vdots & & \ddots & 0 \\
c_{\ell, 0} & \cdots & \cdots & c_{\ell, \ell-1}
\end{array}\right)\left(\begin{array}{c}
1 \\
L_{2} \\
\vdots \\
L_{2}^{\ell-1}
\end{array}\right) .
$$

Here, it is obvious to see that $c_{i, j} \in \mathbb{Q}\left(d_{1}, \cdots, d_{5}\right)$. Noticing the first row, one has

$$
\sum_{i=1}^{\ell} a_{i} f_{i}=c_{1,0} . \quad\left(a_{i} \in \mathbb{Q}\left(d_{1}, \cdots, d_{5}\right)\right) .
$$

Hence, by the definition of $f_{i}$, one gets the desired diffrential equation for $L_{2 \ell}$.

\subsection{Differential equations for $L_{2 \ell-1}$}

A similar procedure developed in the last subsection works also for the odd case. In fact, we have the following.

Proposition 3.4. There exist constants

$$
a_{i}(k), b_{i}(k) \in \mathbb{Q}\left(e_{1}, e_{2}, e_{3}, e_{4}\right) \quad\left(a_{n+k-1}(k) \neq 0, b_{2 n+2 k}(k) \neq 0\right)
$$


such that the functions $L_{2 \ell-1}^{(2 k)}$ and $\left(L_{2 \ell-1}^{(2 k+1)}\right)^{2}$ are expressed by the polynomials of $L_{1}$ as

$$
\begin{aligned}
L_{2 \ell-1}^{(2 k)} & =\sum_{i=0}^{\ell+k-1} a_{i}(k) L_{1}^{2 i+1}, \quad\left(a_{i}(0)=1\right) \\
\left(L_{2 \ell-1}^{(2 k+1)}\right)^{2} & =\sum_{i=0}^{2 \ell+2 k} b_{i}(k) L_{1}^{2 i}
\end{aligned}
$$

Using this proposition one can prove the following result. The proof is the same as Theorem 3.3.

Theorem 3.5. The function $L_{2 \ell-1}(\ell \geq 1)$ satisfies the following differential equation:

$$
\sum_{i=1}^{2 \ell-1} \alpha_{i} L_{2 \ell-1}^{(2 i)} L_{2 \ell-1}^{(8 \ell-4-2 i)}+\sum_{i=0}^{4 \ell-2} \beta_{i} L_{2 \ell-1} L_{2 \ell-1}^{(2 i)}+\gamma=0 \quad\left(\alpha_{i}, \beta_{i}, \gamma \in \mathbb{Q}\left(e_{1}, \cdots, e_{4}\right)\right)
$$

Remark 3.6. One finds that, in the proof of Theorem 3.3, there are certain redundancy in the process for deriving the algebraic differential equation satisfied by $L_{\ell}$. Therefore one can obtain, in principle, much simpler equations, i.e. the number of terms and of derivatives of the equations in Theorem 3.3 and Theorem 3.5 can be reduced.

\section{Examples}

In this section, we give much simpler and explicit differential equations for $L_{4}$ and $L_{3}$ explicitly. These are in fact simpler than what we have given in the preceding section. We use Mathematica 6.

\subsection{A differential equation for $L_{4}$}

By Proposition 3.2 one sees that there exist $a_{i}, b_{i} \in \mathbb{Q}\left(d_{1}, \cdots, d_{5}\right)$ such that

$$
\begin{aligned}
L_{4} L_{4}^{\prime \prime}+a_{1}\left(L_{4}^{\prime}\right)^{2}+a_{2} L_{4}^{(4)}+a_{3} L_{4}^{\prime \prime}+a_{4} L_{4}+a_{5}=b_{1} L_{2}+b_{2}, \\
L_{4} L_{4}^{\prime \prime}+a_{1}\left(L_{4}^{\prime}\right)^{2}+a_{6} L_{4}^{2}+a_{7} L_{4}^{\prime \prime}+a_{8} L_{4}+a_{9}=b_{3} L_{2}+b_{4} .
\end{aligned}
$$

From these equations we obtain

$$
L_{4} L_{4}^{\prime \prime}+a\left(L_{4}^{\prime}\right)^{2}+b L_{4}^{(4)}+c L_{4}^{2}+d L_{4}^{\prime \prime}+e L_{4}+f=0, \quad\left(a, b, c, d, e, f \in \mathbb{Q}\left(d_{1}, \cdots, d_{5}\right)\right) .
$$

By Lemma 3.1 and Lemma 2.2, we have

$$
\begin{aligned}
L_{2}^{\prime \prime} & =d_{2} L_{2}+\frac{6 d_{2}}{d_{1}} L_{4} \\
\left(L_{2}^{\prime}\right)^{2} & =\frac{4 d_{2}}{d_{1}} L_{2}^{3}+d_{2}\left(6 d_{3}+1\right) L_{2}^{2}+4 d_{1} d_{2} d_{4} L_{2}+d_{1}^{2} d_{2} d_{5} .
\end{aligned}
$$

It follows that

$$
\begin{aligned}
L_{2}^{\prime \prime}= & \frac{6 d_{2}}{d_{1}} L_{2}^{2}+d_{2}\left(6 d_{3}+1\right) L_{2}+2 d_{1} d_{2} d_{4} \\
L_{4}= & L_{2}^{2}+d_{1} d_{3} L_{2}+\frac{1}{3} d_{1}^{2} d_{4}, \\
L_{4}^{\prime}= & 2 L_{2} L_{2}^{\prime}+d_{1} d_{3} L_{2}^{\prime}, \\
\left(L_{4}^{\prime}\right)^{2}= & \left(2 L_{2}+d_{1} d_{3}\right)^{2}\left(L_{2}^{\prime}\right)^{2} \\
= & \frac{16 d_{2}}{d_{1}} L_{2}^{5}+4 d_{2}\left(10 d_{3}+1\right) L_{2}^{4}+4 d_{1} d_{2}\left(7 d_{3}^{2}+d_{3}+4 d_{4}\right) L_{2}^{3} \\
& +d_{1}^{2} d_{2}\left(6 d_{3}^{3}+d_{3}^{2}+16 d_{3} d_{4}+4 d_{5}\right) L_{2}^{2}+4 d_{1}^{3} d_{2} d_{3}\left(d_{3} d_{4}+d_{5}\right) L_{2}+d_{1}^{4} d_{2} d_{3}^{2} d_{5},
\end{aligned}
$$




$$
\begin{aligned}
L_{4}^{\prime \prime}= & 2\left(L_{2} L_{2}^{\prime \prime}+\left(L_{2}^{\prime}\right)^{2}\right)+d_{1} d_{3} L_{2}^{\prime \prime} \\
= & \frac{20 d_{2}}{d_{1}} L_{2}^{3}+2 d_{2}\left(15 d_{3}+2\right) L_{2}^{2}+d_{1} d_{2}\left(6 d_{3}^{2}+d_{3}+12 d_{4}\right) L_{2}+2 d_{1}^{2} d_{2}\left(d_{3} d_{4}+d_{5}\right) \\
L_{4}^{\prime \prime \prime}= & {\left[\frac{60 d_{2}}{d_{1}} L_{2}^{2}+4 d_{2}\left(15 d_{3}+2\right) L_{2}+d_{1} d_{2}\left(6 d_{3}^{2}+d_{3}+12 d_{4}\right)\right] L_{2}^{\prime} } \\
L_{4}^{(4)}= & {\left[\frac{60 d_{2}}{d_{1}} L_{2}^{2}+4 d_{2}\left(15 d_{3}+2\right) L_{2}+d_{1} d_{2}\left(6 d_{3}^{2}+d_{3}+12 d_{4}\right)\right] L_{2}^{\prime \prime} } \\
& +\left[\frac{120 d_{2}}{d_{1}} L_{2}+4 d_{2}\left(15 d_{3}+2\right)\right]\left(L_{2}^{\prime}\right)^{2} \\
= & \frac{840 d_{2}^{2}}{d_{1}^{2}} L_{2}^{4}+\frac{20 d_{2}^{2}}{d_{1}}\left(84 d_{3}+13\right) L_{2}^{3}+2 d_{2}^{2}\left(378 d_{3}^{2}+111 d_{3}+336 d_{4}+8\right) L_{2}^{2} \\
& +d_{1} d_{2}^{2}\left(36 d_{3}^{3}+12 d_{3}^{2}+432 d_{3} d_{4}+d_{3}+60 d_{4}+120 d_{5}\right) L_{2} \\
& +2 d_{1}^{2} d_{2}^{2}\left(6 d_{3}^{2} d_{4}+d_{3} d_{4}+12 d_{4}^{2}+30 d_{3} d_{5}+4 d_{5}\right) .
\end{aligned}
$$

Subsituting these into (4.1), we have the linear equation for $a, b, \cdots, f$ as follows:

$$
\left\{\begin{array}{l}
\frac{20 d_{2}}{d_{1}}+a \cdot \frac{16 d_{2}}{d_{1}}=0 \\
2 d_{2}\left(25 d_{3}+2\right)+a \cdot 4 d_{2}\left(10 d_{3}+1\right)+b \cdot \frac{840 d_{2}^{2}}{d_{1}^{2}}+c \cdot 1=0 \\
d_{1} d_{2}\left(36 d_{3}^{2}+5 d_{3}+\frac{56}{3} d_{4}\right)+a \cdot 4 d_{1} d_{2}\left(7 d_{3}^{2}+d_{3}+4 d_{4}\right)+b \cdot \frac{20 d_{2}^{2}}{d_{1}}\left(84 d_{3}+13\right) \\
+c \cdot 2 d_{1} d_{3}+d \cdot \frac{20 d_{2}}{d_{1}}=0 \\
d_{1}^{2} d_{2}\left(6 d_{3}^{3}+d_{3}^{2}+24 d_{3} d_{4}+\frac{4}{3} d_{4}+2 d_{5}\right)+a \cdot d_{1}^{2} d_{2}\left(6 d_{3}^{3}+d_{3}^{2}+16 d_{3} d_{4}+4 d_{5}\right) \\
\quad+b \cdot 2 d_{2}^{2}\left(378 d_{3}^{2}+111 d_{3}+336 d_{4}+8\right)+c \cdot d_{1}^{2}\left(d_{3}^{2}+\frac{2}{3} d_{4}\right)+d \cdot 2 d_{2}\left(15 d_{3}+2\right)+e \cdot 1=0 \\
d_{1}^{3} d_{2}\left(4 d_{3}^{2} d_{4}+\frac{1}{3} d_{3} d_{4}+4 d_{4}^{2}+2 d_{3} d_{5}\right)+a \cdot 4 d_{1}^{3} d_{2} d_{3}\left(d_{3} d_{4}+d_{5}\right) \\
\quad+b \cdot d_{1} d_{2}^{2}\left(36 d_{3}^{3}+12 d_{3}^{2}+432 d_{3} d_{4}+d_{3}+60 d_{4}+120 d_{5}\right)+c \cdot \frac{2}{3} d_{1}^{3} d_{3} d_{4} \\
+d \cdot d_{1} d_{2}\left(6 d_{3}^{2}+d_{3}+12 d_{4}\right)+e \cdot d_{1} d_{3}=0 \\
\frac{2}{3} d_{1}^{4} d_{2} d_{4}\left(d_{3} d_{4}+d_{5}\right)+a \cdot d_{1}^{4} d_{2} d_{3}^{2} d_{5}+b \cdot 2 d_{1}^{2} d_{2}^{2}\left(6 d_{3}^{2} d_{4}+d_{3} d_{4}+12 d_{4}^{2}+30 d_{3} d_{5}+4 d_{5}\right) \\
+c \cdot \frac{1}{9} d_{1}^{4} d_{4}^{2}+d \cdot 2 d_{1}^{2} d_{2}\left(d_{3} d_{4}+d_{5}\right)+e \cdot \frac{1}{3} d_{1}^{2} d_{4}+f \cdot 1=0 .
\end{array}\right.
$$

Solving the equation we can determine the coefficients of the equation (4.1) as follows:

$$
\begin{aligned}
a= & -\frac{5}{4}, \\
b= & \frac{d_{1}^{2}\left(-9 d_{3}^{4}-6 d_{3}^{3}-d_{3}^{2}+24 d_{3}^{2} d_{4}+8 d_{3} d_{4}-16 d_{4}^{2}\right)}{20 d_{2}\left(20 d_{3}^{3}+17 d_{3}^{2}-40 d_{3} d_{4}+4 d_{3}-16 d_{4}+20 d_{5}\right)}, \\
c= & \frac{d_{2}\left(378 d_{3}^{4}+272 d_{3}^{3}+59 d_{3}^{2}-1008 d_{3}^{2} d_{4}-376 d_{3} d_{4}+4 d_{3}+672 d_{4}^{2}-16 d_{4}+20 d_{5}\right)}{20 d_{3}^{3}+17 d_{3}^{2}-40 d_{3} d_{4}+4 d_{3}-16 d_{4}+20 d_{5}}, \\
d= & d_{1}^{2}\left(-12 d_{3}^{5}+36 d_{3}^{4}+40 d_{3}^{3} d_{4}+24 d_{3}^{3}-116 d_{3}^{2} d_{4}+3 d_{3}^{2}-12 d_{3}^{2} d_{5}-32 d_{3} d_{4}^{2}-40 d_{3} d_{4}\right. \\
& \left.-24 d_{3} d_{5}+112 d_{4}^{2}+16 d_{4} d_{5}\right) / 12\left(20 d_{3}^{3}+17 d_{3}^{2}-40 d_{3} d_{4}+4 d_{3}-16 d_{4}+20 d_{5}\right), \\
e & d_{1}^{2} d_{2}\left(1332 d_{3}^{6}-48 d_{3}^{5}-549 d_{3}^{4}-156 d_{3}^{3}-12 d_{3}^{2}-5328 d_{3}^{4} d_{4}-1472 d_{3}^{3} d_{4}-104 d_{3}^{2} d_{4}\right. \\
& -64 d_{3} d_{4}+2304 d_{3}^{2} d_{4}^{2}+448 d_{3} d_{4}^{2}+448 d_{4}^{2}+5376 d_{4}^{3}+7200 d_{3}^{3} d_{5}+6000 d_{3}^{2} d_{5} \\
& \left.+1200 d_{3} d_{5}-14400 d_{3} d_{4} d_{5}-5600 d_{4} d_{5}+3600 d_{5}^{2}\right) / 60\left(20 d_{3}^{3}+17 d_{3}^{2}-40 d_{3} d_{4}\right. \\
& \left.+4 d_{3}-16 d_{4}+20 d_{5}\right), \\
f= & d_{1}^{4} d_{2}\left(12 d_{3}^{2} d_{4}+84 d_{3}^{3} d_{4}+45 d_{3}^{4} d_{4}-222 d_{3}^{5} d_{4}-16 d_{3} d_{4}^{2}-1244 d_{3}^{2} d_{4}^{2}-6608 d_{3}^{3} d_{4}^{2}\right. \\
& -11280 d_{3}^{4} d_{4}^{2}-128 d_{4}^{3}+8032 d_{3} d_{4}^{3}+29760 d_{3}^{2} d_{4}^{3}-15360 d_{4}^{4}-18 d_{3}^{2} d_{5}+1152 d_{3}^{3} d_{5} \\
& +6633 d_{3}^{4} d_{5}+9720 d_{3}^{5} d_{5}-2016 d_{3} d_{4} d_{5}-17568 d_{3}^{2} d_{4} d_{5}-37200 d_{3}^{3} d_{4} d_{5}+8752 d_{4}^{2} d_{5} \\
& \left.+30720 d_{3} d_{4}^{2} d_{5}+720 d_{3} d_{5}^{2}+4860 d_{3}^{2} d_{5}^{2}-11280 d_{4} d_{5}^{2}\right) \\
& / 180\left(20 d_{3}^{3}+17 d_{3}^{2}-40 d_{3} d_{4}+4 d_{3}-16 d_{4}+20 d_{5}\right) .
\end{aligned}
$$




\subsection{A differential equation for $L_{3}$}

The following differential equation satisfied by $L_{3}$ can be obtained by a similar discussion made in the case for $L_{4}$.

$$
L_{3}^{2} L_{3}^{\prime \prime}+a L_{3}\left(L_{3}^{\prime}\right)^{2}+b L_{3}^{3}+c L_{3}^{(6)}+d L_{3}(4)+e L_{3}^{\prime \prime}+f L_{3}=0, \quad\left(a, b, c, d, e, f \in \mathbb{Q}\left(e_{1}, \cdots, e_{4}\right)\right) .
$$

The coefficients appeared in the equation above can be calculated as follows:

$$
\begin{aligned}
& a=-\frac{4}{3}, \\
& b=e_{2}\left(-37494 e_{3}+16758 e_{1}^{2} e_{3}-210291 e_{3}^{2}+100275 e_{1}^{2} e_{3}^{2}+1216848 e_{3}^{3}-770844 e_{1}^{2} e_{3}^{3}\right. \\
& +10259968 e_{3}^{4}-4157814 e_{1}^{2} e_{3}^{4}+17720640 e_{3}^{5}-834840 e_{1}^{2} e_{3}^{5}+224964 e_{4} \\
& -100548 e_{1}^{2} e_{4}+1365696 e_{3} e_{4}+286272 e_{1}^{2} e_{3} e_{4}-5531328 e_{3}^{2} e_{4}+4352904 e_{1}^{2} e_{3}^{2} e_{4} \\
& -24675840 e_{3}^{3} e_{4}-11950560 e_{1}^{2} e_{3}^{3} e_{4}-4209408 e_{4}^{2}-2612736 e_{1}^{2} e_{4}^{2}-22256640 e_{3} e_{4}^{2} \\
& \left.+17418240 e_{1}^{2} e_{3} e_{4}^{2}\right) /\left(1 2 \left(-4166 e_{3}+1862 e_{1}^{2} e_{3}-36803 e_{3}^{2}+23555 e_{1}^{2} e_{3}^{2}-124484 e_{3}^{3}\right.\right. \\
& +71384 e_{1}^{2} e_{3}^{3}-154224 e_{3}^{4}+13914 e_{1}^{2} e_{3}^{4}+24996 e_{4}-11172 e_{1}^{2} e_{4}+146352 e_{3} e_{4} \\
& \left.\left.-42672 e_{1}^{2} e_{3} e_{4}+88704 e_{3}^{2} e_{4}+199176 e_{1}^{2} e_{3}^{2} e_{4}+48384 e_{4}^{2}-290304 e_{1}^{2} e_{4}^{2}\right)\right), \\
& c=e_{1}^{3}\left(72 e_{3}^{2}-72 e_{3}^{3}-2332 e_{3}^{4}-4725 e_{3}^{5}-864 e_{3} e_{4}-1368 e_{3}^{2} e_{4}+10800 e_{3}^{3} e_{4}+2592 e_{4}^{2}\right. \\
& \left.+10800 e_{3} e_{4}^{2}\right) /\left(1 3 5 e _ { 2 } ^ { 2 } \left(-4166 e_{3}+1862 e_{1}^{2} e_{3}-36803 e_{3}^{2}+23555 e_{1}^{2} e_{3}^{2}-124484 e_{3}^{3}\right.\right. \\
& +71384 e_{1}^{2} e_{3}^{3}-154224 e_{3}^{4}+13914 e_{1}^{2} e_{3}^{4}+24996 e_{4}-11172 e_{1}^{2} e_{4}+146352 e_{3} e_{4} \\
& \left.\left.-42672 e_{1}^{2} e_{3} e_{4}+88704 e_{3}^{2} e_{4}+199176 e_{1}^{2} e_{3}^{2} e_{4}+48384 e_{4}^{2}-290304 e_{1}^{2} e_{4}^{2}\right)\right) \text {, } \\
& d=e_{1}^{3}\left(546 e_{3}^{2}-5586 e_{1}^{2} e_{3}^{2}+43207 e_{3}^{3}-35287 e_{1}^{2} e_{3}^{3}+61307 e_{3}^{4}+233393 e_{1}^{2} e_{3}^{4}\right. \\
& -1118174 e_{3}^{5}+1314554 e_{1}^{2} e_{3}^{5}-2930256 e_{3}^{6}+264366 e_{1}^{2} e_{3}^{6}-6552 e_{3} e_{4}+67032 e_{1}^{2} e_{3} e_{4} \\
& -399498 e_{3}^{2} e_{4}+339738 e_{1}^{2} e_{3}^{2} e_{4}-1518936 e_{3}^{3} e_{4}-123384 e_{1}^{2} e_{3}^{3} e_{4}-1090656 e_{3}^{4} e_{4} \\
& +4034796 e_{1}^{2} e_{3}^{4} e_{4}+19656 e_{4}^{2}-201096 e_{1}^{2} e_{4}^{2}+696384 e_{3} e_{4}^{2}+102816 e_{1}^{2} e_{3} e_{4}^{2} \\
& \left.+2515968 e_{3}^{2} e_{4}^{2}-1930608 e_{1}^{2} e_{3}^{2} e_{4}^{2}+870912 e_{4}^{3}-5225472 e_{1}^{2} e_{4}^{3}\right) /\left(1080 e_{2}\right. \\
& \left(-4166 e_{3}+1862 e_{1}^{2} e_{3}-36803 e_{3}^{2}+23555 e_{1}^{2} e_{3}^{2}-124484 e_{3}^{3}+71384 e_{1}^{2} e_{3}^{3}\right. \\
& -154224 e_{3}^{4}+13914 e_{1}^{2} e_{3}^{4}+24996 e_{4}-11172 e_{1}^{2} e_{4}+146352 e_{3} e_{4}-42672 e_{1}^{2} e_{3} e_{4} \\
& \left.\left.+88704 e_{3}^{2} e_{4}+199176 e_{1}^{2} e_{3}^{2} e_{4}+48384 e_{4}^{2}-290304 e_{1}^{2} e_{4}^{2}\right)\right) \text {, } \\
& e=-e_{1}^{3}\left(9282 e_{3}^{2}-27930 e_{1}^{2} e_{3}^{2}+272819 e_{3}^{3}-288155 e_{1}^{2} e_{3}^{3}+635939 e_{3}^{4}+628805 e_{1}^{2} e_{3}^{4}\right. \\
& -6674273 e_{3}^{5}+11265830 e_{1}^{2} e_{3}^{5}-26757152 e_{3}^{6}+16964470 e_{1}^{2} e_{3}^{6}-19640880 e_{3}^{7} \\
& +3061080 e_{1}^{2} e_{3}^{7}-111384 e_{3} e_{4}+335160 e_{1}^{2} e_{3} e_{4}-1251066 e_{3}^{2} e_{4}+1698690 e_{1}^{2} e_{3}^{2} e_{4} \\
& -6930792 e_{3}^{3} e_{4}+2736360 e_{1}^{2} e_{3}^{3} e_{4}-82630752 e_{3}^{4} e_{4}+80842140 e_{1}^{2} e_{3}^{4} e_{4} \\
& -234924480 e_{3}^{5} e_{4}+58289280 e_{1}^{2} e_{3}^{5} e_{4}+334152 e_{4}^{2}-1005480 e_{1}^{2} e_{4}^{2}-4782672 e_{3} e_{4}^{2} \\
& +4536000 e_{1}^{2} e_{3} e_{4}^{2}-60303744 e_{3}^{2} e_{4}^{2}+23127120 e_{1}^{2} e_{3}^{2} e_{4}^{2}-133781760 e_{3}^{3} e_{4}^{2} \\
& +169248960 e_{1}^{2} e_{3}^{3} e_{4}^{2}+14805504 e_{4}^{3}-26127360 e_{1}^{2} e_{4}^{3}+26127360 e_{3} e_{4}^{3} \\
& \left.+104509440 e_{1}^{2} e_{3} e_{4}^{3}\right) /\left(2 1 6 0 \left(-4166 e_{3}+1862 e_{1}^{2} e_{3}-36803 e_{3}^{2}+23555 e_{1}^{2} e_{3}^{2}\right.\right. \\
& -124484 e_{3}^{3}+71384 e_{1}^{2} e_{3}^{3}-154224 e_{3}^{4}+13914 e_{1}^{2} e_{3}^{4}+24996 e_{4}-11172 e_{1}^{2} e_{4} \\
& \left.\left.+146352 e_{3} e_{4}-42672 e_{1}^{2} e_{3} e_{4}+88704 e_{3}^{2} e_{4}+199176 e_{1}^{2} e_{3}^{2} e_{4}+48384 e_{4}^{2}-290304 e_{1}^{2} e_{4}^{2}\right)\right), \\
& f=e_{1}^{3} e_{2}\left(5958 e_{3}^{2}-16758 e_{1}^{2} e_{3}^{2}+190245 e_{3}^{3}-239925 e_{1}^{2} e_{3}^{3}+1012737 e_{3}^{4}-303117 e_{1}^{2} e_{3}^{4}\right. \\
& -2753838 e_{3}^{5}+8314998 e_{1}^{2} e_{3}^{5}-31889088 e_{3}^{6}+36513498 e_{1}^{2} e_{3}^{6}-70418016 e_{3}^{7} \\
& +39563376 e_{1}^{2} e_{3}^{7}-36546048 e_{3}^{8}+6752928 e_{1}^{2} e_{3}^{8}-71496 e_{3} e_{4}+201096 e_{1}^{2} e_{3} e_{4} \\
& -1108926 e_{3}^{2} e_{4}+2091726 e_{1}^{2} e_{3}^{2} e_{4}-29384904 e_{3}^{3} e_{4}+16327704 e_{1}^{2} e_{3}^{3} e_{4}
\end{aligned}
$$




$$
\begin{aligned}
& -140392064 e_{3}^{4} e_{4}+89458724 e_{1}^{2} e_{3}^{4} e_{4}+33067264 e_{3}^{5} e_{4}-43487104 e_{1}^{2} e_{3}^{5} e_{4} \\
& +763931136 e_{3}^{6} e_{4}+72474944 e_{1}^{2} e_{3}^{6} e_{4}+214488 e_{4}^{2}-603288 e_{1}^{2} e_{4}^{2}+41926464 e_{3} e_{4}^{2} \\
& -46345824 e_{1}^{2} e_{3} e_{4}^{2}+351313920 e_{3}^{2} e_{4}^{2}-124364880 e_{1}^{2} e_{3}^{2} e_{4}^{2}-1274706432 e_{3}^{3} e_{4}^{2} \\
& +2125418112 e_{1}^{2} e_{3}^{3} e_{4}^{2}-6904461312 e_{3}^{4} e_{4}^{2}-131194368 e_{1}^{2} e_{3}^{4} e_{4}^{2}-252730368 e_{4}^{3} \\
& +254596608 e_{1}^{2} e_{4}^{3}-2993614848 e_{3} e_{4}^{3}+990517248 e_{1}^{2} e_{3} e_{4}^{3}-7851810816 e_{3}^{2} e_{4}^{3} \\
& \left.+6524292096 e_{1}^{2} e_{3}^{2} e_{4}^{3}-1170505728 e_{4}^{4}+7023034368 e_{1}^{2} e_{4}^{4}\right) /\left(5 7 6 0 \left(-4166 e_{3}\right.\right. \\
& +1862 e_{1}^{2} e_{3}-36803 e_{3}^{2}+23555 e_{1}^{2} e_{3}^{2}-124484 e_{3}^{3}+71384 e_{1}^{2} e_{3}^{3}-154224 e_{3}^{4} \\
& +13914 e_{1}^{2} e_{3}^{4}+24996 e_{4}-11172 e_{1}^{2} e_{4}+146352 e_{3} e_{4}-42672 e_{1}^{2} e_{3} e_{4} \\
& \left.\left.+88704 e_{3}^{2} e_{4}+199176 e_{1}^{2} e_{3}^{2} e_{4}+48384 e_{4}^{2}-290304 e_{1}^{2} e_{4}^{2}\right)\right) .
\end{aligned}
$$

Corrigenda to the paper $[\mathrm{KW}]$ : We list here some corrections of the equations in the paper [KW]. We did not, however, use these equations.

- The coefficient of the second term of (2.12) in Corollary 2.2 is incorrect and should be 48 .

- The coefficient of $B_{3}(q)$ in the expression of $\beta_{2}$ in Theorem 2.3 is incorrect and should be $-\frac{16}{3}$.

- The equation (2.30) in p.37 should be read as $M_{2}^{\prime \prime}(x)=M_{2}(x)\left(6 \beta_{0}^{2} L_{1}(x)^{2}+\beta_{1}\right)$. Hence, the correct equation (2.32) in Corollary 2.4 (p.38) is

$$
M_{2}^{\prime \prime}(x)^{2}+4 \beta_{1} M_{2}(x) M_{2}^{\prime \prime}(x)+\left(36 \beta_{0}^{2} \beta_{2}-5 \beta_{1}^{2}\right) M_{2}(x)^{2}-36 \beta_{0}^{4} M_{2}(x)^{4}=0 .
$$

\section{References}

[C] R. Chapman, Private communication, 2006.

[KW] N. Kurokawa and M. Wakayama, Certain family of elliptic functions defined by q-series, The Ramnujan J. 10 (2005), 23-41.

[L] S. Lang, "Elliptic Functions", Addison Wesley, 1970.

\section{MAsATo WAKAYAMA}

Faculty of Mathematics, Kyushu University.

Hakozaki Higashi-ku, Fukuoka 812-8581, JAPAN.

wakayama@math.kyushu-u.ac.jp

\section{KeITARO YAMAMOTO}

Graduate School of Mathematics, Kyushu University.

Hakozaki Higashi-ku, Fukuoka, 812-8581 JAPAN.

Current address:

JASTEC Co.,Ltd.

3-5-23, Takanawa, Minato-ku, Tokyo 108-0074 JAPAN 


\section{List of MI Preprint Series, Kyushu University}

The Grobal COE Program

Math-for-Industry Education \& Research Hub

MI

MI2008-1 Takahiro ITO, Shuichi INOKUCHI \& Yoshihiro MIZOGUCHI

Abstract collision systems simulated by cellular automata

MI2008-2 Eiji ONODERA

The intial value problem for a third-order dispersive flow into compact almost

Hermitian manifolds

MI2008-3 Hiroaki KIDO

On isosceles sets in the 4-dimensional Euclidean space

MI2008-4 Hirofumi NOTSU

Numerical computations of cavity flow problems by a pressure stabilized characteristiccurve finite element scheme

MI2008-5 Yoshiyasu OZEKI

Torsion points of abelian varieties with values in nfinite extensions over a padic field

MI2008-6 Yoshiyuki TOMIYAMA

Lifting Galois representations over arbitrary number fields

MI2008-7 Takehiro HIROTSU \& Setsuo TANIGUCHI

The random walk model revisited

MI2008-8 Silvia GANDY, Masaaki KANNO, Hirokazu ANAI \& Kazuhiro YOKOYAMA Optimizing a particular real root of a polynomial by a special cylindrical algebraic decomposition

MI2008-9 Kazufumi KIMOTO, Sho MATSUMOTO \& Masato WAKAYAMA

Alpha-determinant cyclic modules and Jacobi polynomials 
MI2008-10 Sangyeol LEE \& Hiroki MASUDA

Jarque-Bera Normality Test for the Driving Lévy Process of a Discretely Observed Univariate SDE

MI2008-11 Hiroyuki CHIHARA \& Eiji ONODERA

A third order dispersive flow for closed curves into almost Hermitian manifolds

MI2008-12 Takehiko KINOSHITA, Kouji HASHIMOTO and Mitsuhiro T. NAKAO

On the $L^{2}$ a priori error estimates to the finite element solution of elliptic problems with singular adjoint operator

MI2008-13 Jacques FARAUT and Masato WAKAYAMA

Hermitian symmetric spaces of tube type and multivariate Meixner-Pollaczek polynomials

MI2008-14 Takashi NAKAMURA

Riemann zeta-values, Euler polynomials and the best constant of Sobolev inequality

MI2008-15 Takashi NAKAMURA

Some topics related to Hurwitz-Lerch zeta functions

MI2009-1 Yasuhide FUKUMOTO

Global time evolution of viscous vortex rings

MI2009-2 Hidetoshi MATSUI \& Sadanori KONISHI

Regularized functional regression modeling for functional response and predictors

MI2009-3 Hidetoshi MATSUI \& Sadanori KONISHI

Variable selection for functional regression model via the $L_{1}$ regularization

MI2009-4 Shuichi KAWANO \& Sadanori KONISHI

Nonlinear logistic discrimination via regularized Gaussian basis expansions

MI2009-5 Toshiro HIRANOUCHI \& Yuichiro TAGUCHII

Flat modules and Groebner bases over truncated discrete valuation rings 
MI2009-6 Kenji KAJIWARA \& Yasuhiro OHTA

Bilinearization and Casorati determinant solutions to non-autonomous $1+1$ dimensional discrete soliton equations

\section{MI2009-7 Yoshiyuki KAGEI}

Asymptotic behavior of solutions of the compressible Navier-Stokes equation around the plane Couette flow

MI2009-8 Shohei TATEISHI, Hidetoshi MATSUI \& Sadanori KONISHI

Nonlinear regression modeling via the lasso-type regularization

MI2009-9 Takeshi TAKAISHI \& Masato KIMURA

Phase field model for mode III crack growth in two dimensional elasticity

MI2009-10 Shingo SAITO

Generalisation of Mack's formula for claims reserving with arbitrary exponents for the variance assumption

MI2009-11 Kenji KAJIWARA, Masanobu KANEKO, Atsushi NOBE \& Teruhisa TSUDA Ultradiscretization of a solvable two-dimensional chaotic map associated with the Hesse cubic curve

\section{MI2009-12 Tetsu MASUDA}

Hypergeometric T -functions of the q-Painlevé system of type $E_{8}^{(1)}$

MI2009-13 Hidenao IWANE, Hitoshi YANAMI, Hirokazu ANAI \& Kazuhiro YOKOYAMA A Practical Implementation of a Symbolic-Numeric Cylindrical Algebraic Decomposition for Quantifier Elimination

MI2009-14 Yasunori MAEKAWA

On Gaussian decay estimates of solutions to some linear elliptic equations and its applications

MI2009-15 Yuya ISHIHARA \& Yoshiyuki KAGEI

Large time behavior of the semigroup on $L^{p}$ spaces associated with the linearized compressible Navier-Stokes equation in a cylindrical domain 
MI2009-16 Chikashi ARITA, Atsuo KUNIBA, Kazumitsu SAKAI \& Tsuyoshi SAWABE Spectrum in multi-species asymmetric simple exclusion process on a ring

MI2009-17 Masato WAKAYAMA \& Keitaro YAMAMOTO

Non-linear algebraic differential equations satisfied by certain family of elliptic functions 